\title{
Problems and Countermeasures of Government Affairs Openness-Take Shandong Province as an example
}

\author{
Wei Wang ${ }^{1,3, ~ a ~, ~ Y i k a i ~ L i a n g ~}{ }^{2,4, ~ b, ~}{ }^{*}, \mathrm{Ke} \mathrm{He}^{4, \mathrm{c}}$ and Yiming Liu 1, 3, d \\ ${ }^{1}$ Shandong Computer Science Center (National Supercomputer Center in Jinan), Shandong Provincial Key \\ Laboratory of Computer Networks, Jinan, 250101, China \\ ${ }^{2}$ School of Management Science and Engineering, Shandong University of Finance and Economics, Jinan \\ 250014, China \\ ${ }^{3}$ Shandong Institute of Economy and Informatization Development, Jinan, 250014, China \\ ${ }^{4}$ School of Management, Shandong University, Jinan 250100, China \\ awangw@sdas.org, byikailiang@qq.com, c594241423@qq.com, diuym@sdas.org \\ *corresponding author
}

Keywords: Government affairs openness, evaluation, provincial government, municipal government.

\begin{abstract}
Government affairs openness is of great significance for the government to setup a reasonable and efficient government service. Leaders at all levels attach great importance to it, and relevant policies have also been introduced. In response to the national call, to further promote open government, improve government transparency, this paper uses the investigation method, taking Shandong Province as an example, with the help of computing center of Shandong province (the National Supercomputing Center in Jinan), we organized open assessment working group to assess the Shandong provincial government departments and the municipal government in 2016 and analyzed the result. Then, we find the problems existing in the present stage, and put forward some suggestions for improvement.
\end{abstract}

\section{Introduction}

Public affairs mainly refers to the work of government public, the work for the government planning or preparing for the classification of the public, the work content and process to the public, any citizen can carry out query and supervision through specific ways, such as open data, open network and so on [1]. Openness of government affairs plays an important role in promoting administration according to law, promoting the transformation of government functions, improving administrative efficiency, preventing and controlling corruption [2]. In the course of more than 20 years' practice, the openness of government affairs in our country has not only accumulated rich experience, but also appeared the situation of lower legislative level and unreasonable mechanism, which needs further improvement [3]. At present, the Shandong provincial government has responded positively to the call of the state, and has made great improvements in government work, but there are still many problems. At the same time, there are many deficiencies in the research of the government affairs openness, and lack of targeted research. This paper takes Shandong Province (SD) as an example, evaluate and analysis the government affairs openness of SD in 2016, to explore the present situation of Shandong government, and found the public problems, put forward some suggestions for improvement.It will bring beneficial enlightenment for the government to effectively promote the government affairs openness. 


\section{Research Methods}

The purpose of this study is to explore the current situation of the openness of government affairs in SD and find out the existing problems. Using survey method to collect can get some information that difficult to obtain from direct observation, not restricted by time and space, and has high efficiency, can get a lot of data in a short period of time, at the same time to promote the work of relevant units itself through investigation, and it is widely used in the research on the current problem. Therefore, this article adopts the survey method to evaluate and analyze the government affairs of the relevant departments in SD in 2016.

The assessment target is 42 provincial government departments (including constituent sectors, departments that directly under the ad hoc agencies, institutions and departments directly under the Administration) and 17 municipal governments.

The evaluation index includes four first level indicators: "Openness of administrative power operation", "Information openness in key fields", "Government involvement and response concerns" and "Capacity building of government affairs openness". The corresponding weights were 30\%, 25\%, 25\%, and 20\%. In addition, the four first level indexes contains 16 second level indexes, covers six aspects of Public Affairs (the management of public services, public policy implementation, the people's livelihood, public power, public policy response, system and platform construction).

\section{Results Analysis}

\subsection{The evaluation of government affairs openness about provincial government departments}

According to figure 1, the average score of provincial government departments is 72.1844 , the distribution is generally normal distribution, the overall work of government affairs openness is in the steady progress stage. The general index average score index was $71.56 \%$, the average score of special index was $76.64 \%$. The general indicators score index of Provincial Economic and Information Commission, the Provincial Department of culture, food and Drug Supervision Bureau, the provincial Environmental Protection Bureau and the Provincial Quality Supervision Bureau of is all higher than 80\%; for the special indicators, the special indicators score index of the provincial food and drug administration, the Provincial Work Safety Supervision Bureau, the Provincial Department of culture, the provincial Price Bureau, the provincial human resources and social security department Province, Industrial and Commercial Bureau score index reached more than $90 \%$. In the 42 provincial government departments of this evaluation, the Provincial Department of culture ranked first, provincial economic and Information Commission, the provincial food and Drug Supervision Bureau, the Provincial Quality Supervision Bureau, the provincial Environmental Protection Bureau, the provincial SASAC, the provincial development and Reform Commission, the provincial forestry department, the provincial Industrial and Commercial Bureau and the Provincial Department of human resources and social security in second to tenth.

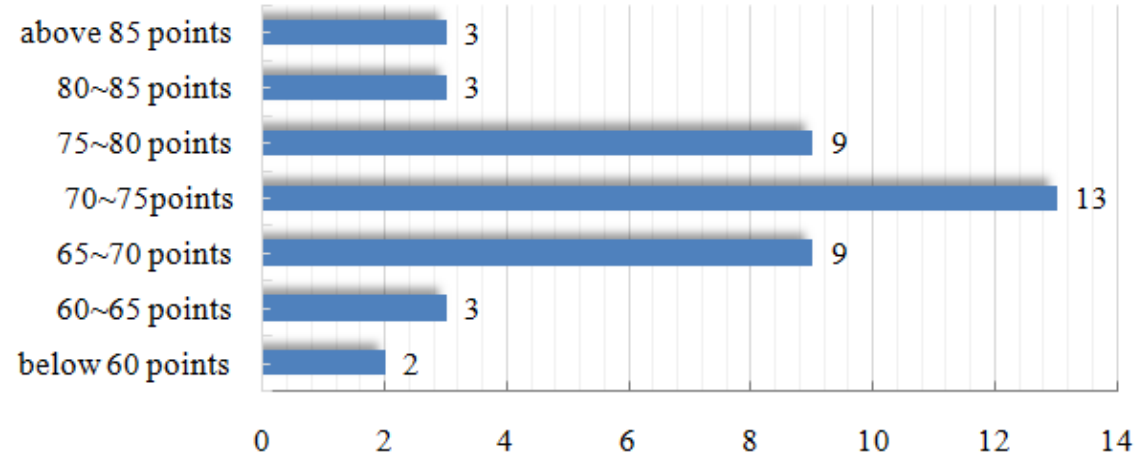

Fig. 1 the distribution of assessment scores 


\subsection{The evaluation of government affairs openness about municipal government departments}

From the score of the indicators, the government of the 17 cities at the corresponding level of government affairs works well (as shown in figure 2). The average assessment score of the 34 county-level government openness is 61.12 , basically reached the passing standard, but the county government level of public affairs is uneven, polarization is serious. Among them, the score index of Weihai City and Weifang City in "administrative power operation" reached $94.83 \%$ and 89.17\%, ahead of other municipal government; as for "information openness in key fields", Weifang City, Jinan City, Weihai city and Dezhou City, the performance is more prominent; the score index of Weihai City, Dezhou city and Weifang city in "government involvement and response concerns" is higher than 88.30\%; Weihai City, Zaozhuang City, Qingdao City and Tai'an City have a high level in "capacity building of government affairs openness", the score index reached $95 \%$ or more. Of the 17 municipal governments evaluated, Weihai ranked first, Weifang, Dezhou, Tai'an and Linyi ranked second to fifth.

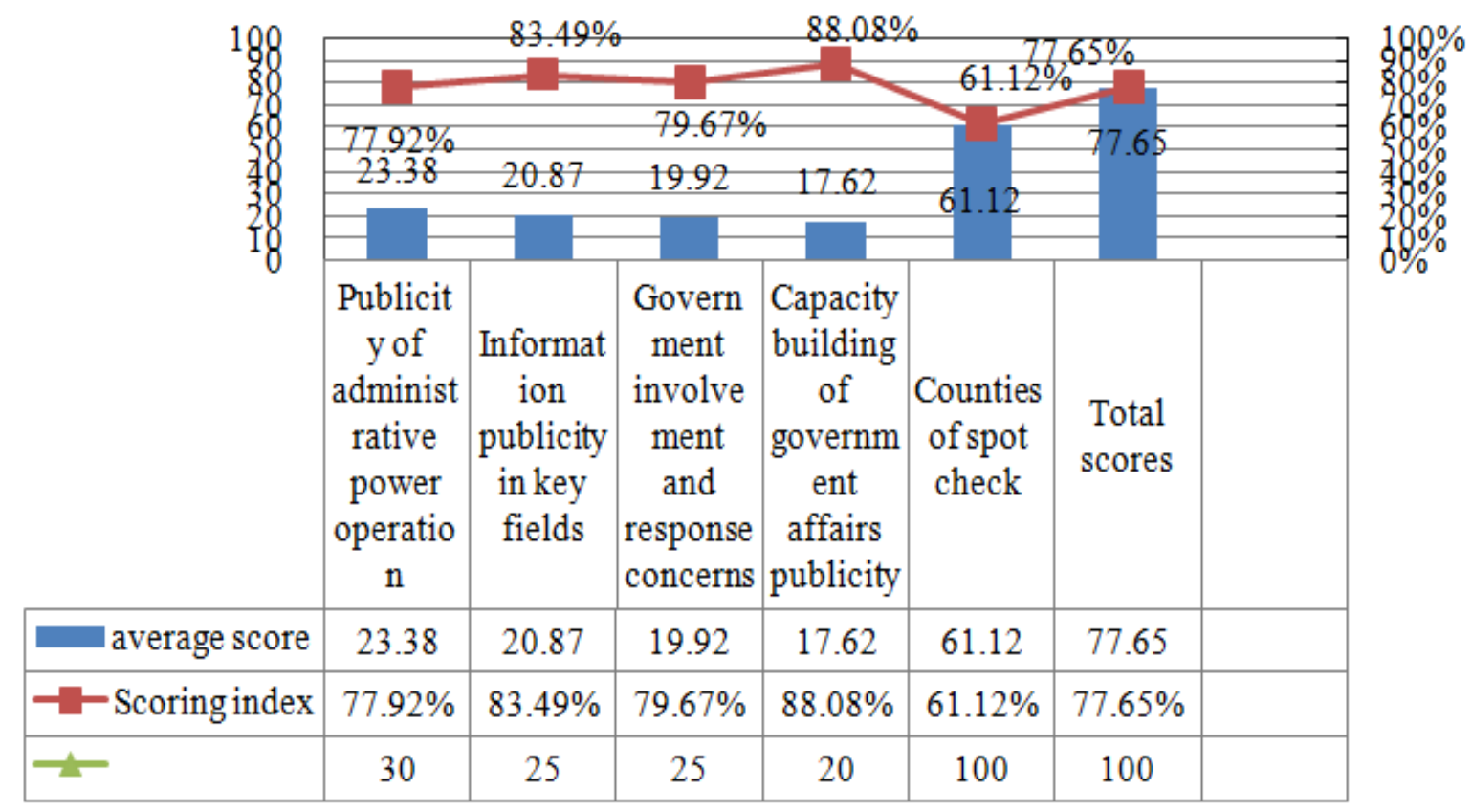

Fig. 2 Average assessment of municipal government departments

Through the result of assessment, this study summarizes the situation of the Shandong provincial government in government affairs openness as follows.

\subsubsection{The openness of power operation level improved significantly, "five openness" effect highlights}

All units are able to open the draft decision and decision basis before the decision, 30 provincial government departments and 16 municipal government through the portal comment column provides online channels to collect opinions before making a decision, the remaining units also disclosed the draft decision and decision basis in the announcement column; all units are actively explore the establishment of relevant interests party government conference system, the government released important meetings and activities information through the government website, expanding public participation.

\subsubsection{Information disclosure in key areas is generally better, and the scope of information disclosure in people's livelihood areas is gradually expanding}

As for the financial budget and final accounts, the provincial government departments and municipalities have publicly announced the financial accounts in 2015 and the budget of 2016, public content is more refined. The scope of information disclosure in people's livelihood areas has 
gradually expanded. For the poverty alleviation, 16 municipal government public poverty alleviation policies, poverty alleviation projects and implementation, as well as poverty exit information on the portals in different forms. For the social assistance, Weihai, Weifang, Zaozhuang and other 16 municipal governments classified relief openness information in the region generally in the form of columns. For the information disclosure of employment and entrepreneurship, the 16 municipal governments announced the employment and entrepreneurship policies, timely updates market supply and demand information. As for the renovation of dilapidated buildings in rural areas and affordable housing information disclosure, 16 municipal governments disclosed shantytowns information in the region, 14 municipal governments discloses rural reconstruction information in the region, all the 17 municipal government public affordable housing information in the region.

\subsubsection{Policy interpretation gradually normalized, and respond to concerns become more actively and timely}

All units set up a policy interpretation column in the portal, more than $30 \%$ of the units' responsible person can attend the press conference, receive an interview or publish articles to take the lead to preach policies; policy interpretation form gradually diversified. Most of the units are arranged in the column that can respond to hot portals, in response to the social hot issues and important public opinion, $81 \%$ of the provincial government departments, $82 \%$ municipal government and $76 \%$ of the county government have opened online interview column, 27 provincial departments, 17 municipal government and 27 county government public leadership mailbox.

\subsubsection{The ability to open government affairs has been obviously improved, and the mechanism system has been gradually improved}

The platform of government affairs openness are diversified, and all provincial government departments, municipal governments and county governments can provide comprehensive search in the portal website. Among them, the retrieval function on websites of 40 provincial government departments and 16 municipal governments is better, and the retrieval results are quick and accurate. On the other hand, 19 provincial government departments and 17 municipal governments have also set up news conference columns at the portal. At the end of January 2017, there are 39 provincial government departments opened a government micro-blog, accounted for 92.86\%; 17 municipal governments all launched; and 28 county-level government opened micro-blog, accounting for 82.35\%.

\section{Main problems of government affairs openness}

Through the analysis of the evaluation results above, this study finds that the following problems exist in the government affairs openness of SD.

\subsection{Collection and practical applications of public opinion need to be improved}

Most units can carry out online opinions collection and public opinion surveys center on major decisions, draft of normative documents and concerns of the public. However, after the end of the consultation, $71 \%$ of the provincial government departments and $70 \%$ of the municipal government were unable to timely publish the situation of opinion collection, the adoption and the reasons for not adopted. Some units carry out less number of activities in the government website, online survey subjects of 31\% provincial government departments and 29\% municipal government departments are only limited to web page revision, website satisfaction, lack of major livelihood decisions polls. 


\subsection{The depth and quality of information openness in some key fields need to be strengthened}

Some of the key areas of information disclosure still have unclear open standards, content is not refined enough, for example, government investment in major construction projects are mostly news information, the elements of information content is incomplete. In the field of livelihood information, some units adopt domain classification, which makes it very inconvenient for the public to obtain information. Part of the city's key areas of information disclosure directory directly linked to the relevant departments of the city's home page, it is not conducive to inquire required information, the depth and quality of openness needs to be strengthened [4].

\subsection{The interpretation of local policy is not adequate}

In the interpretation of local policy, some units still have the problem that only use a single form, lack of digital, Q\&A, charts, illustrations, audio, video and other means. There are policy interpretation column of some units, mainly reproduced by the relevant departments of the state or higher authorities, lack of the interpretation of the policy in the region. The interpretation of the information issued by the provincial government departments, which is closely related to the macro economy and the people's livelihood, is not ideal enough.

\section{Suggestions for the future work of government affairs openness}

In view of the problems discovered, this paper puts forward the following suggestions for the government affairs of SD.

\subsection{Exploring the standardization of government affairs openness}

The evaluation shows that the overall level and standardization of government affairs in the county government of SD are relatively low, and the development is uneven and polarization is serious. It is recommended that the provincial government office and the Provincial Quality Supervision Bureau should in the province to take the lead in promoting standardization of government affairs, promoting the depth of integration of government affairs and standardization work. Selecting a county (city, district) as a pilot to explore the way to the openness of basic characteristics, and gradually improve our province public affairs of local standards, provide the basic contents and methods of public work to follow for all the levels of government. On the basis of the first trial, we should strive for 5 counties (cities, districts) of our province to become standardized pilot of standardized government affairs at the national level [5].

\subsection{Re-planning provincial government information disclosure platform}

The provincial government public information platform has been used as a unified platform for the integration of provincial government portal and for the government to release the information in various regions and departments. In the comprehensive promotion of public affairs under the new situation, its development needs to be re-planned. It is recommended that the provincial government office and public affairs departments should organize experts and representatives of the public to do some research and demonstration on the future development direction of the provincial government information disclosure platform, make reasonable planning, clear its role and position. Establishing the joint update mechanism of provincial government information disclosure platform and various departments and regional portal information disclosure column, to achieve the "one point of release, multi point sharing", and solve the problem of untimely artificial regeneration which lead to inconsistent information.

\subsection{Further strengthening policy interpretation}

It is suggested that the general office of the provincial government should introduce policies and interpret relevant policies or standards, making clear provisions for the scope, time limit, form and content of the departments at various levels, so as to further standardize the interpretation of 
policies. To establish the synchronization mechanism of policy interpretation and policy formulation work, policy documents and interpretation program, interpretation of materials should be synchronized organization, synchronization signing and synchronous deployment.

\section{References}

[1] Kassen, M. (2013). A promising phenomenon of open data: a case study of the Chicago open data project. Government Information Quarterly, 30(4), 508-513.

[2] Mcdermott, P. (2010). Building open government. Government Information Quarterly, 27(4), 401-413.

[3] Attard, J., Orlandi, F., Scerri, S., \& Auer, S. (2015). A systematic review of open government data initiatives. Government Information Quarterly, 32(4), 399-418.

[4] Conradie, P., \& Choenni, S. (2014). On the barriers for local government releasing open data. Government Information Quarterly, 31, S10-S17.

[5] Yang, T. M., \& Wu, Y. J. (2016). Examining the socio-technical determinants influencing government agencies' open data publication: a study in Taiwan. Government Information Quarterly, 33(3), 378-392. 